\title{
Systemic Immunotherapy for the Treatment of Brain Metastases
}

\author{
Justine V. Cohen* and Harriet M. Kluger \\ Section of Medical Oncology, Department of Medicine, Yale Cancer Center, New Haven, CT, USA
}

Keywords: immunotherapy, brain metastases, melanoma

\section{BACKGROUND}

Significant progress has been made in the treatment of selected malignancies with immunemodulating antibodies. Phase III trials of anti-CTLA-4 in melanoma and anti-PD-1 in melanoma, renal cell carcinoma (RCC), and non-small cell lung cancer (NSCLC) showed improved overall survival (OS) compared to standard therapies (1-5). As a result, immune checkpoint inhibitors are now approved for the treatment of these diseases. Blockade of CTLA-4 (ipilumimab and tremelimumab), PD-1 (nivolumab, pembrolizumab, pidilizumab and others), and PD-L1 [BMS 936559 (6), durvalimumab (7), and atezolizumabes (8-11)] can produce durable responses in patients with metastatic cancer. Clinical trials with these agents, alone and in combination, are ongoing. Moreover, additional immune checkpoint modulators are in pre-clinical and clinical development. Other approved immunotherapies include high-dose bolus interleukin-2 (IL-2), interferon alpha-2b, and Sipuleucel-T. There are limited data, however, on the impact of immunotherapy in patients with measurable metastatic disease to the brain. Registration trials of immune therapies excluded patients with active brain metastases based on a historical poor prognosis in this patient population coupled with uncertainty about the ability of the drugs to cross the blood brain barrier (BBB). These active therapies might however have benefited patients with microscopic brain deposits.

Brain metastases were historically managed with whole brain radiation therapy (WBRT) or surgical resection, depending on the size, number, histology, symptoms, and location. The availability of high-resolution magnetic resonance imaging (MRI) and stereotactic radiosurgery (SRS) to small, emerging lesions has improved local lesional control. These modalities allow higher doses of radiation. In many institutions, WBRT is reserved for patients with multiple or larger lesions not amenable to SRS $(12,13)$. These treatments are not without limitations and consequences. For example, WBRT has been associated with cognitive decline, while SRS can result in radiation necrosis, cerebral edema, and delayed tumor hemorrhage $(14,15)$. More often, however, focal therapies are limited in efficacy due to distant cerebral relapse and lack of treatment of microscopic tumor foci not evident on imaging. As new systemic treatments, particularly immune-modulating agents, show prolonged survival of patients with aggressive extra-cerebral disease, these drugs need to be assessed for efficacy in active brain metastases. There are a number of ongoing investigations to determine if these antibodies cross the leaky BBB found in tumors despite their size $(16,17)$. Alternatively, although brain metastases might contain pre-existing tumor infiltrating lymphocytes (TILs), immune modulation induced by these agents may allow cytotoxic $\mathrm{T}$ cells into the tumor microenvironment in the brain, resulting in antitumor immunity. Several lines of evidence suggest that $\mathrm{T}$ cells within the tumor microenvironment are responsible for the responses seen with these therapies $(18,19)$. To date, there have been no published pharmacokinetic or pharmacodynamic studies in on-treatment brain tissue to allow determination of drug penetration into the tumor, primarily due to the difficulty accruing patients to trials requiring brain biopsies, particularly from patients who are responding to therapy. Although animal studies have been done, drug distribution and $\mathrm{T}$ cell activation might not reflect that of humans. 
Metastatic melanoma is the solid tumor with the highest propensity for dissemination to the brain (20). The only chemotherapy widely used for melanoma known to definitively cross $\mathrm{BBB}$ is temozolamide, which induced responses in $7 \%$ of melanoma brain metastasis patients (21). Other anti-neoplastic drugs that cross the $\mathrm{BBB}$ include fotemustine, etoposide, cisplatin, vinblastine, and motoxantrone and can be used depending on tumor cell sensitivity (22-26). Targeted therapies such as erlotinib, afatinib, and lapatinib have also shown evidence of ability to cross the BBB (27-29).

\section{PRECLINICAL DATA}

The ability of immune-modulating antibodies to cross the BBB and control brain metastases is the subject of ongoing investigations. In primary CNS tumors, preclinical data with immunemodulating antibodies have shown promise. In mice with SMA-650 intracranial tumors, anti-CTLA-4 was tolerated well (30). An increase in CD4+ cells and decrease in T regs prolonged survival in these animals. Similarly, PD-1 blockade combined with radiation was tested in mice with GL261 intracranial tumors and showed improved survival (31). The combination of PD-1 and CTLA-4 inhibitors similarly showed improved survival in animal models (32). These examples suggest that BBB drug penetration in tumors might be obtainable, for primary CNS tumors and for metastatic tumors, although this remains to be verified in humans with each drug and tumor type.

\section{CLINICAL DATA}

High-dose IL-2 was one of the first immune-modulating agents to demonstrate activity in melanoma and RCC. There have not been any formal trials of IL-2 specifically for patients with brain metastases. A retrospective series reported a response rate in active brain metastases lower than expected for extra-cerebral disease, however without excessive toxicities (33). One of the first studies to investigate the effect of immunotherapy on brain metastases in patients with metastatic melanoma was a retrospective analysis of the phase II trial with ipilimumab, which reported 5 of 12 patients were responders $(34,35)$. Following this observation, a phase II trial of ipilimumab specifically for patients with brain metastases from melanoma opened (36). Results of 72 patients accrued showed prolonged OS, particularly notable in asymptomatic patients. These findings were confirmed in an expanded access protocol of ipilimumab with a $20 \% 1$-year OS in patients with stable, asymptomatic brain metastases (37). Based on these promising results, the Italian Network for Tumor Biotherapy (NIBIT) designed a phase II trial of ipilimumab in combination with fotemustine (NIBIT-M1) with twenty asymptomatic patients with brain metastases. Stable disease or partial response was seen in $25 \%$ and another $25 \%$ had complete response in the brain $(38,39)$.

A follow-up randomized trial (NIBIT-M2) was subsequently initiated for patients with untreated melanoma brain metastases comparing fotemustine monotherapy, fotemustine plus ipilimumab $10 \mathrm{mg} / \mathrm{kg}$ and ipilimumab $3 \mathrm{mg} / \mathrm{kg}$ + nivolumab $10 \mathrm{mg} /$ $\mathrm{kg}$ (NCT02460068). Objectives include OS, safety, disease control rate (intra and extra-cerebral) objective response rate, duration of response, and progression-free survival. This study will also examine quality of life. Various groups are studying the effect of immune-modulating agents alone and in combination with other therapies for the treatment of brain metastases from melanoma. For example, ipilimumab and nivolumab or nivolumab monotherapy is being studied in a large multi-arm phase II trial (NCT02320058 and NCT02374242) and combinations of ipilimumab with various forms and schedules of radiation are being investigated (NCT01703507, NCT01950195 and NCT02097732). Results of these trials are pending.

A phase II trial of pembrolizumab for patients with metastatic melanoma or NSCLC and untreated brain metastases is ongoing. Preliminary results from this trial were presented at ASCO 2015 (NCT02085070) (40, 41). In this two-arm study, patients are eligible if they have at least 1 untreated or progressive brain metastasis $(5-20 \mathrm{~mm})$, not requiring steroids and are without neurological symptoms. Patients in the melanoma arm require brain metastasis biopsy or resection of metastatic brain lesion prior to starting therapy or availability of previously resected brain lesions for correlative studies. Patients in the NSCLC arm are required to have PD-L1 positive tumors. In the NSCLC arm, 11 patients were evaluable for response as of June 2015. Brain metastasis response rate was $45 \%$, and systemic response rate was $45 \%$. Only one patient with a systemic response had disease progression in the brain, and two patients with disease progression as their best systemic response were unevaluable in the brain due to rapid systemic progression. The duration of response in the brain was at least 12 weeks for four of five responders, and all responses were ongoing at the time of data analysis (40). In the melanoma arm, 18 patients were accrued at the time of analysis. Four patients were unevaluable due to rapid extra-cerebral progression or hemorrhage, and one was too early for response evaluation. Four patients achieved partial response, three had stable disease, and seven had disease progression (two with mixed response and one with histologically demonstrated pseudoprogression). Response in the body was largely concordant with brain response, although in some cases brain response occurred after extracerebral response. Response in the brain was ongoing at $4+, 6+, 6+$, and $11+$ months (41).

Studies completed to date suggest that immune checkpoint inhibitors have activity in the brain that might be similar to that of extra-cerebral sites (42). In the phase II study of ipilimumab brain metastases activity in asymptomatic patients was similar to that of patients without brain metastases with a disease control rate of 24 and 27\%, respectively. The 1- and 2-year progression-free survival were 31 and $26 \%$, respectively $(36,43)$. The NIBIT-M1 study described above confirmed these findings with an immunerelated disease control rate for patients with brain metastases of $50 \%$ compared with $46.5 \%$ of the entire treated population. Interim data from our phase II trial of pembrolizumab in patients with metastatic melanoma and NSCLC with untreated brain metastases showed that all responses in the melanoma arm were concordant, while three or four in the NSCLC arm were concordant $(40,41)$. Results suggest that immune-modulating agents may have similar durable responses in the brain as seen systemically, and support use of systemic therapy alone or in combination with 
focal therapy (SRS or surgery) in the treatment of brain metastases from immune therapy responsive diseases such as melanoma and lung cancer.

There are data to suggest that responses might be further improved by combining immune checkpoint inhibitors with radiation. Several studies have evaluated the combination in other disease sites (44-47). A number of mechanisms have been described explaining the combined effect; radiation upregulates inflammatory cytokines (i.e., TNF $\alpha$, IFN- $\gamma$, and CXCL16), promoting tumor detection and facilitating $\mathrm{T}$ cell infiltration (48, 49). Radiation can upregulate PD-L1 (50). The abscopal effect, in which local radiation is thought to cause a systemic response resulting in shrinkage at distant sites, further supports the use of radiation combined with immune-modulating agents (51). Knisely et al. published a series of patients with metastatic melanoma with brain metastases who achieved a median survival of 21.3 months if they received ipilimumab and SRS versus 4.9 months if they underwent SRS but did not receive ipilimumab (44). Mathew et al. looked at a similar population with 25 patients receiving both ipilimumab and SRS versus 33 patients receiving SRS alone (46). The analysis did not show a significant benefit in 6-month OS between the two groups, although this was not a randomized trial and the groups were not balanced. Lastly, Silk et al. reported improved OS in patients receiving ipilimumab and SRS (47). Exploratory analysis within the same study showed no increase in OS with the addition of ipilimumab to WBRT. The timing of administration of concurrent immune checkpoint inhibitors and radiation has not yet been determined. Kiess et al. found increased rates of progression if patients were treated with SRS before or during ipilimumab compared with those who received SRS after systemic therapy (52). Future studies will provide insight into the optimal timing for combining radiation and immune-modulating therapies, such as NCT02097732, which is investigating SRS to brain metastases before or in the middle of ipilimumab induction.

Toxicities unique to central nervous system metastases, such as vasogenic edema and tumor necrosis represent an additional challenge. Early recognition of potential symptoms is essential. One of the challenges in treating brain metastasis patients with immune therapy is management of neurological symptoms, which might be from perilesional edema, intralesional hemorrhage, necrosis most commonly seen in previously irradiated lesions, or tumor growth due to treatment failure. Examples of perilesional edema seen on FLAIR images before and on therapy in two patients receiving pembrolizumab are shown in Figure 1. Both patients responded well to transient steroids and remain on pembrolizumab with good disease control for over a year. Depending on the size and location of the brain metastasis, patients might require surgical intervention due to neurologic symptoms. Moreover, it is sometimes impossible to determine whether lesions enlarge on study due to inflammation, necrosis, or tumor growth, and current imaging modalities can be inadequate (53). Our institutional experience suggests that despite the indisputable benefit of systemic immune therapy in some tumor types, radiation necrosis occurs with greater frequency in patients treated with immunotherapy than other types of systemic therapy. We, and others, have used bevacizumab to control perilesional

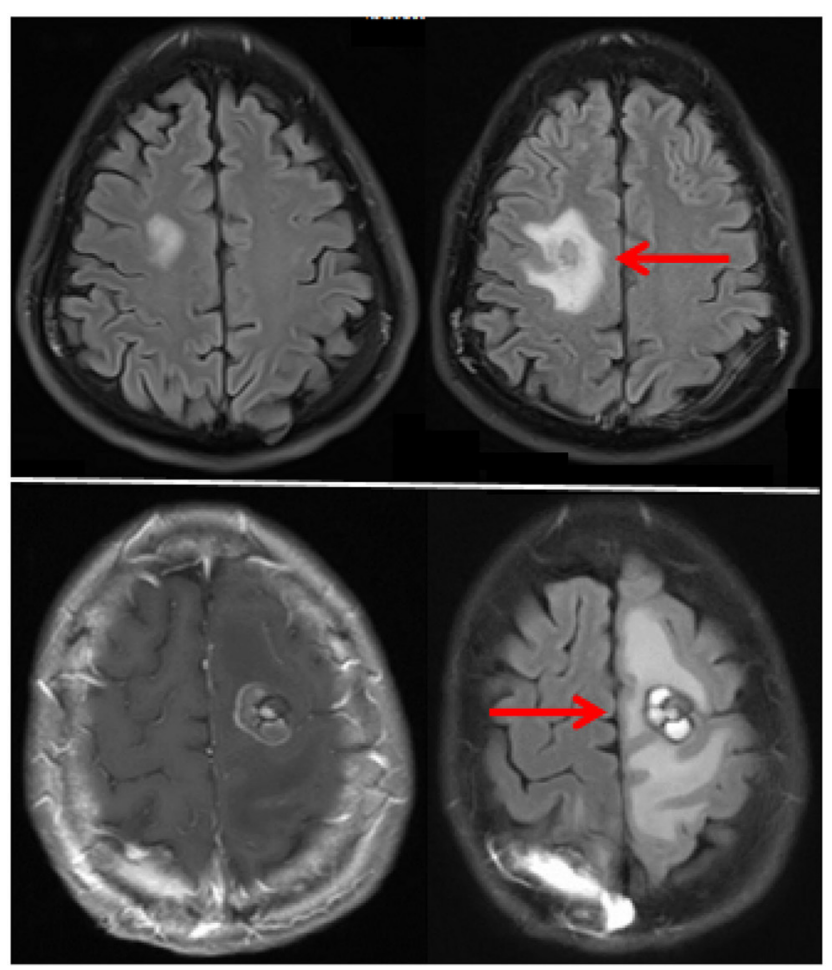

FIGURE 1 | MRI FLAIR images of two patients with perilesional while receiving pembrolizumab. The top and bottom frames represent the two separate patients. Images prior to therapy are on the left and after therapy on the right.

edema and worsening radiation necrosis, with variable success, and surgical intervention or laser interstitial thermacoagulation therapy is sometimes needed although caution must be taken with histologies more prone to hemorrhage (54-59). Furthermore, the incidence of seizures from perilesional edema might be decreased with use of prophylactic anti-epileptic medications.

\section{FUTURE DIRECTIONS AND CONCLUSION}

Use of immune therapy for non-irradiated brain metastases has shown promise in a small number of clinical trials, and requires validation in larger studies and in different tumor types. Experience to date suggests that activity of immune checkpoint inhibitors in brain metastases is similar to that of extracerebral metastases, and exclusion of patients with brain metastases from clinical investigations is no longer justified, although separate studies or separate cohorts for patients with untreated brain metastases might be required. Challenges with treating this patient population include drug-related toxicities such as perilesional edema and tumor-related confounding factors such as necrosis in previously irradiated lesions and intralesional hemorrhage, both of which might require intervention with local or systemic modalities such as surgery, radiation, anticonvulsants, steroids, or VEGF inhibitors. Efficacy of immune checkpoint inhibitors might be further enhanced by combining more than one inhibitor 
or with combinations with chemotherapy, targeted therapy, or radiation therapy. As the breadth of immunotherapies available for investigation and use expands, predictive biomarkers will also need to be studied and validated. This can be particularly challenging in patients with brain metastases due to the morbidity associated with biopsy; however, if concordance of response is persistently observed as newer drugs are studied in this patient population, extra-cerebral biopsies might suffice. Clinical trials

\section{REFERENCES}

1. Garon EB, Rizvi NA, Hui R, Leighl N, Balmanoukian AS, Eder JP, et al. Pembrolizumab for the treatment of non-small-cell lung cancer. N Engl J Med (2015) 372(21):2018-28. doi:10.1056/NEJMoa1501824

2. Paz-Ares L, Horn L, Borghaei H, Spigel DR, Steins M, Ready N, et al. Phase III, randomized trial (CheckMate 057) of nivolumab (NIVO) versus docetaxel (DOC) in advanced non-squamous cell (non-SQ) non-small cell lung cancer (NSCLC). ASCO Meeting Abstracts (2015) 33(15_suppl):LBA109.

3. Weber JS, D’Angelo SP, Minor D, Hodi FS, Gutzmer R, Neyns B, et al. Nivolumab versus chemotherapy in patients with advanced melanoma who progressed after anti-CTLA-4 treatment (CheckMate 037): a randomised, controlled, open-label, phase 3 trial. Lancet Oncol (2015) 16(4):375-84. doi:10.1016/S1470-2045(15)70076-8

4. Motzer RJ, Escudier B, McDermott DF, George S, Hammers HJ, Srinivas S, et al. Nivolumab versus everolimus in advanced renal-cell carcinoma. $N$ Engl $J$ Med (2015) 373(19):1803-13. doi:10.1056/NEJMoa1510665

5. McDermott D, Haanen J, Chen TT, Lorigan P, O'Day S, Investigators MDX. Efficacy and safety of ipilimumab in metastatic melanoma patients surviving more than 2 years following treatment in a phase III trial (MDX010-20). Ann Oncol (2013) 24(10):2694-8. doi:10.1093/annonc/mdt291

6. Brahmer JR, Tykodi SS, Chow LQ, Hwu WJ, Topalian SL, Hwu P, et al. Safety and activity of anti-PD-L1 antibody in patients with advanced cancer. $N$ Engl $J$ Med (2012) 366(26):2455-65. doi:10.1056/NEJMoa1200694

7. Brahmer JR, Rizvi NA, Lutzky J, Khleif S, Blake-Haskins A, Li X, et al. Clinical activity and biomarkers of MEDI4736, an anti-PD-L1 antibody, in patients with NSCLC. ASCO Meeting Abstracts (2014) 32(15_suppl):8021.

8. Powles T, Eder JP, Fine GD, Braiteh FS, Loriot Y, Cruz C, et al. MPDL3280A (anti-PD-L1) treatment leads to clinical activity in metastatic bladder cancer. Nature (2014) 515(7528):558-62. doi:10.1038/nature13904

9. Hamid O, Sosman JA, Lawrence DP, Sullivan RJ, Ibrahim N, Kluger HM, et al. Clinical activity, safety, and biomarkers of MPDL3280A, an engineered PD-L1 antibody in patients with locally advanced or metastatic melanoma (mM). ASCO Meeting Abstracts (2013) 31(15_suppl):9010.

10. Herbst RS, Soria JC, Kowanetz M, Fine GD, Hamid O, Gordon MS, et al. Predictive correlates of response to the anti-PD-L1 antibody MPDL3280A in cancer patients. Nature (2014) 515(7528):563-7. doi:10.1038/nature14011

11. Tabernero J, Powderly JD, Hamid O, Gordon MS, Fisher GA, Braiteh FS, et al. Clinical activity, safety, and biomarkers of MPDL3280A, an engineered PD-L1 antibody in patients with locally advanced or metastatic CRC, gastric cancer (GC), SCCHN, or other tumors. ASCO Meeting Abstracts (2013) 31(15_suppl):3622.

12. Staudt M, Lasithiotakis K, Leiter U, Meier F, Eigentler T, Bamberg M, et al. Determinants of survival in patients with brain metastases from cutaneous melanoma. Br J Cancer (2010) 102(8):1213-8. doi:10.1038/sj.bjc.6605622

13. Mathieu D, Kondziolka D, Cooper PB, Flickinger JC, Niranjan A, Agarwala S, et al. Gamma knife radiosurgery for malignant melanoma brain metastases. Clin Neurosurg (2007) 54:241-7.

14. Redmond AJ, Diluna ML, Hebert R, Moliterno JA, Desai R, Knisely JP, et al. Gamma knife surgery for the treatment of melanoma metastases: the effect of intratumoral hemorrhage on survival. J Neurosurg (2008) 109(Suppl):99-105. doi:10.3171/JNS/2008/109/12/S16

15. Brown PD, Asher AL, Ballman KV, Farace E, Cerhan JH, Anderson SK, et al. NCCTG N0574 (alliance): a phase III randomized trial of whole brain radiation therapy (WBRT) in addition to radiosurgery (SRS) in patients with 1 to 3 brain metastases. ASCO Meeting Abstracts (2015) 33(15_suppl):LBA4. designed specifically for this patient population addressing the effects of multi-modality therapy, particularly combinations of immune checkpoint inhibitors and radiation, are necessary for improving outcomes among individuals with brain metastases.

\section{AUTHOR CONTRIBUTIONS}

JC and HK wrote this article together.

16. Di Giacomo AM, Margolin K. Immune checkpoint blockade in patients with melanoma metastatic to the brain. Semin Oncol (2015) 42(3):459-65. doi:10.1053/j.seminoncol.2015.02.006

17. Pestalozzi BC, Brignoli S. Trastuzumab in CSF. JClin Oncol (2000) 18(11):2349-51.

18. Wilson EH, Weninger W, Hunter CA. Trafficking of immune cells in the central nervous system. J Clin Invest (2010) 120(5):1368-79. doi:10.1172/JCI41911

19. Engelhardt B, Coisne C. Fluids and barriers of the CNS establish immune privilege by confining immune surveillance to a two-walled castle moat surrounding the CNS castle. Fluids Barriers CNS (2011) 8(1):4. doi:10.1186/2045-8118-8-4

20. Flanigan JC, Jilaveanu LB, Faries M, Sznol M, Ariyan S, Yu JB, et al. Melanoma brain metastases: is it time to reassess the bias? Curr Probl Cancer (2011) 35(4):200-10. doi:10.1016/j.currproblcancer.2011.07.003

21. Agarwala SS, Kirkwood JM, Gore M, Dreno B, Thatcher N, Czarnetski B, et al. Temozolomide for the treatment of brain metastases associated with metastatic melanoma: a phase II study. J Clin Oncol (2004) 22(11):2101-7. doi:10.1200/JCO.2004.11.044

22. Avril MF, Aamdal S, Grob JJ, Hauschild A, Mohr P, Bonerandi JJ, et al. Fotemustine compared with dacarbazine in patients with disseminated malignant melanoma: a phase III study. J Clin Oncol (2004) 22(6):1118-25. doi:10.1200/JCO.2004.04.165

23. Green RM, Stewart DJ, Hugenholtz H, Richard MT, Thibault M, Montpetit V. Human central nervous system and plasma pharmacology of mitoxantrone. J Neurooncol (1988) 6(1):75-83. doi:10.1007/BF00163544

24. Stewart DJ, Leavens M, Maor M, Feun L, Luna M, Bonura J, et al. Human central nervous system distribution of cis-diamminedichloroplatinum and use as a radiosensitizer in malignant brain tumors. Cancer Res (1982) 42(6):2474-9.

25. Stewart DJ, Lu K, Benjamin RS, Leavens ME, Luna M, Yap HY, et al. Concentration of vinblastine in human intracerebral tumor and other tissues. J Neurooncol (1983) 1(2):139-44.

26. Stewart DJ, Richard MT, Hugenholtz H, Dennery JM, Belanger R, GerinLajoie J, et al. Penetration of VP-16 (etoposide) into human intracerebral and extracerebral tumors. J Neurooncol (1984) 2(2):133-9.

27. Bai H, Han B. The effectiveness of erlotinib against brain metastases in non-small cell lung cancer patients. Am J Clin Oncol (2013) 36(2):110-5. doi:10.1097/COC.0b013e3182438c91

28. Hata A, Katakami N. Afatinib for erlotinib refractory brain metastases in a patient with EGFR-mutant non-small-cell lung cancer: can high-affinity TKI substitute for high-dose TKI? J Thorac Oncol (2015) 10(7):e65-6. doi:10.1097/ JTO.0000000000000479

29. Saleem A, Searle GE, Kenny LM, Huiban M, Kozlowski K, Waldman AD, et al. Lapatinib access into normal brain and brain metastases in patients with Her-2 overexpressing breast cancer. EJNMMI Res (2015) 5:30. doi:10.1186/ s13550-015-0103-5

30. Fecci PE, Ochiai H, Mitchell DA, Grossi PM, Sweeney AE, Archer GE, et al. Systemic CTLA-4 blockade ameliorates glioma-induced changes to the CD4+ $\mathrm{T}$ cell compartment without affecting regulatory T-cell function. Clin Cancer Res (2007) 13(7):2158-67. doi:10.1158/1078-0432.CCR-06-2070

31. Zeng J, See AP, Phallen J, Jackson CM, Belcaid Z, Ruzevick J, et al. Anti-PD-1 blockade and stereotactic radiation produce long-term survival in mice with intracranial gliomas. Int J Radiat Oncol Biol Phys (2013) 86(2):343-9. doi:10.1016/j.ijrobp.2012.12.025

32. Reardon DA, Gokhale PC, Klein SR, Ligon KL, Rodig SJ, Ramkissoon SH, et al. Glioblastoma eradication following immune checkpoint blockade in an orthotopic, immunocompetent model. Cancer Immunol Res (2016) 4(2):124-35. doi:10.1158/2326-6066.CIR-15-0151 
33. Guirguis LM, Yang JC, White DE, Steinberg SM, Liewehr DJ, Rosenberg SA, et al. Safety and efficacy of high-dose interleukin-2 therapy in patients with brain metastases. JImmunother (2002) 25(1):82-7. doi:10.1097/00002371-200201000-00009

34. Weber JS, Amin A, Minor D, Siegel J, Berman D, O’Day SJ. Safety and clinical activity of ipilimumab in melanoma patients with brain metastases: retrospective analysis of data from a phase 2 trial. Melanoma Res (2011) 21(6):530-4. doi:10.1097/CMR.0b013e32834d3d88

35. Weber J, Thompson JA, Hamid O, Minor D, Amin A, Ron I, et al. A randomized, double-blind, placebo-controlled, phase II study comparing the tolerability and efficacy of ipilimumab administered with or without prophylactic budesonide in patients with unresectable stage III or IV melanoma. Clin Cancer Res (2009) 15(17):5591-8. doi:10.1158/1078-0432.CCR-09-1024

36. Margolin K, Ernstoff MS, Hamid O, Lawrence D, McDermott D, Puzanov I, et al. Ipilimumab in patients with melanoma and brain metastases: an open-label, phase 2 trial. Lancet Oncol (2012) 13(5):459-65. doi:10.1016/ S1470-2045(12)70090-6

37. Heller KN, Pavlick AC, Hodi FS, Thompson JA, Margolin KA, Lawrence DP, et al. Safety and survival analysis of ipilimumab therapy in patients with stable asymptomatic brain metastases. ASCO Meeting Abstracts (2011) 29(15_suppl):8581.

38. Di Giacomo AM, Ascierto PA, Pilla L, Santinami M, Ferrucci PF, Giannarelli $\mathrm{D}$, et al. Ipilimumab and fotemustine in patients with advanced melanoma (NIBIT-M1): an open-label, single-arm phase 2 trial. Lancet Oncol (2012) 13(9):879-86. doi:10.1016/S1470-2045(12)70324-8

39. Di Giacomo AM, Ascierto PA, Queirolo P, Pilla L, Ridolfi R, Santinami M, et al. Three-year follow-up of advanced melanoma patients who received ipilimumab plus fotemustine in the Italian network for tumor biotherapy (NIBIT)-M1 phase II study. Ann Oncol (2015) 26(4):798-803. doi:10.1093/ annonc/mdu577

40. Goldberg SB, Gettinger SN, Mahajan A, Herbst RS, Chiang AC, Tsiouris AJ, et al. Activity and safety of pembrolizumab in patients with metastatic non-small cell lung cancer with untreated brain metastases. ASCO Meeting Abstracts (2015) 33(15_suppl):8035.

41. Kluger HM, Goldberg SB, Sznol M, Tsiouris J, Vortmeyer A, Jilaveanu L, et al. Safety and activity of pembrolizumab in melanoma patients with untreated brain metastases. ASCO Meeting Abstracts (2015) 33(15_suppl):9009.

42. Yushak ML, Chiang VL, Kluger HM. Clinical trials in melanoma patients with brain metastases. Pigment Cell Melanoma Res (2015) 28(6):741-3. doi:10.1111/pcmr.12401

43. Hodi FS, O'Day SJ, McDermott DF, Weber RW, Sosman JA, Haanen JB, et al. Improved survival with ipilimumab in patients with metastatic melanoma. $N$ Engl J Med (2010) 363(8):711-23. doi:10.1056/NEJMoa1003466

44. Knisely JP, Yu JB, Flanigan J, Sznol M, Kluger HM, Chiang VL. Radiosurgery for melanoma brain metastases in the ipilimumab era and the possibility of longer survival. J Neurosurg (2012) 117(2):227-33. doi:10.3171/2012.5.JNS111929

45. Patel KR, Lawson DH, Kudchadkar RR, Carthon BC, Oliver DE, OkwanDuodu $\mathrm{D}$, et al. Two heads better than one? Ipilimumab immunotherapy and radiation therapy for melanoma brain metastases. Neuro Oncol (2015) 17(10):1312-21. doi:10.1093/neuonc/nov093

46. Mathew M, Tam M, Ott PA, Pavlick AC, Rush SC, Donahue BR, et al. Ipilimumab in melanoma with limited brain metastases treated with stereotactic radiosurgery. Melanoma Res (2013) 23(3):191-5. doi:10.1097/ CMR.0b013e32835f3d90
47. Silk AW, Bassetti MF, West BT, Tsien CI, Lao CD. Ipilimumab and radiation therapy for melanoma brain metastases. Cancer Med (2013) 2(6):899-906. doi:10.1002/cam4.140

48. Frey B, Rubner Y, Kulzer L, Werthmoller N, Weiss EM, Fietkau R, et al. Antitumor immune responses induced by ionizing irradiation and further immune stimulation. Cancer Immunol Immunother (2014) 63(1):29-36. doi:10.1007/s00262-013-1474-y

49. Lee Y, Auh SL, Wang Y, Burnette B, Wang Y, Meng Y, et al. Therapeutic effects of ablative radiation on local tumor require CD8+ T cells: changing strategies for cancer treatment. Blood (2009) 114(3):589-95. doi:10.1182/ blood-2009-02-206870

50. Twyman-Saint Victor C, Rech AJ, Maity A, Rengan R, Pauken KE, Stelekati E, et al. Radiation and dual checkpoint blockade activate non-redundant immune mechanisms in cancer. Nature (2015) 520(7547):373-7. doi:10.1038/nature14292

51. Postow MA, Callahan MK, Barker CA, Yamada Y, Yuan J, Kitano S, et al. Immunologic correlates of the abscopal effect in a patient with melanoma. $N$ Engl J Med (2012) 366(10):925-31. doi:10.1056/NEJMoa1112824

52. Kiess AP, Wolchok JD, Barker CA, Postow MA, Tabar V, Huse JT, et al. Stereotactic radiosurgery for melanoma brain metastases in patients receiving ipilimumab: safety profile and efficacy of combined treatment. Int J Radiat Oncol Biol Phys (2015) 92(2):368-75. doi:10.1016/j.ijrobp.2015.01.004

53. Colaco RJ, Martin P, Kluger HM, Yu JB, Chiang VL. Does immunotherapy increase the rate of radiation necrosis after radiosurgical treatment of brain metastases? J Neurosurg (2015):1-7. doi:10.3171/2015.6.JNS142763

54. Torres-Reveron J, Tomasiewicz HC, Shetty A, Amankulor NM, Chiang VL. Stereotactic laser induced thermotherapy (LITT): a novel treatment for brain lesions regrowing after radiosurgery. J Neurooncol (2013) 113(3):495-503. doi:10.1007/s11060-013-1142-2

55. Socinski MA, Langer CJ, Huang JE, Kolb MM, Compton P, Wang L, et al. Safety of bevacizumab in patients with non-small-cell lung cancer and brain metastases. J Clin Oncol (2009) 27(31):5255-61. doi:10.1200/JCO.2009.22.0616

56. De Braganca KC, Janjigian YY, Azzoli CG, Kris MG, Pietanza MC, Nolan $\mathrm{CP}$, et al. Efficacy and safety of bevacizumab in active brain metastases from non-small cell lung cancer. J Neurooncol (2010) 100(3):443-7. doi:10.1007/ s11060-010-0200-2

57. Zustovich F, Ferro A, Farina P. Bevacizumab as first-line therapy for patients with brain metastases from renal carcinoma: a case series. Clin Genitourin Cancer (2014) 12(3):e107-10. doi:10.1016/j.clgc.2013.12.005

58. Zustovich F, Ferro A, Lombardi G, Farina P, Zagonel V. Bevacizumab-based therapy for patients with brain metastases from non-small-cell lung cancer: preliminary results. Chemotherapy (2014) 60(5-6):294-9. doi:10.1159/000376605

59. Zustovich F, Ferro A, Lombardi G, Zagonel V, Fiduccia P, Farina P. Bevacizumab as front-line treatment of brain metastases from solid tumors: a case series. Anticancer Res (2013) 33(9):4061-5.

Conflict of Interest Statement: The trial of Pembrolizumab in patients with brain metastases from melanoma or non-small cell lung cancer was sponsored by the Yale Cancer Center; partial financial support was provided by Merck, Sharp and Dohme.

Copyright $\odot 2016$ Cohen and Kluger. This is an open-access article distributed under the terms of the Creative Commons Attribution License (CC BY). The use, distribution or reproduction in other forums is permitted, provided the original author(s) or licensor are credited and that the original publication in this journal is cited, in accordance with accepted academic practice. No use, distribution or reproduction is permitted which does not comply with these terms. 